\title{
PROBING TOWARD THE SOLID STATE PLASMA ACCELERATOR FRONTIER USING CHANNELING RADIATION MEASUREMENTS AT THE FERMILAB AO PHOTOINJECTOR
}

\author{
R. A. CARRIGAN, JR., \\ Fermi National Accelerator Laboratory, Batavia, Illinois 60510, USA*
}

J. FREUDENBERGER, S. FRITZLER, H. GENZ, A. RICHTER, A. USHAKOV, AND A. ZILGES

Institut für Kernphysik, Technische Universität Darmstadt, Schlossgartenstrasse 9, D-64289

Darmstadt, Germany**

\section{J. P. F. SELLSCHOP}

Schonland Centre, University of the Witwatersrand, 2050 Johannesburg, South Africa

\begin{abstract}
Plasmas offer the possibility of high acceleration gradients. An intriguing suggestion is to use the higher plasma densities possible in solids to get extremely high gradients. Although solid state plasmas might produce high gradients they would pose daunting problems. Crystal channeling has been suggested as one mechanism to address these challenges. There is no experimental or theoretical guidance on channeling in intense electron and laser beams. A high density plasma in a crystal lattice could quench the channeling process. An experiment is being carried out at the Fermilab A0 Photo-Injector Test Facility to observe electron channeling radiation at high bunch charges. An electron beam with up to $8 \mathrm{nC}$ per electron bunch has been used to investigate the electron-crystal interaction. No evidence has been found of significant quenching of channeling at charge densities several orders of magnitude larger than in earlier experiments.
\end{abstract}

\section{Introduction}

Recently there has been interesting progress in studies of plasma acceleration in gasses [1]. This has been due in part to the development of terawatt laser technology [2] a decade ago. Gas plasmas have already delivered gradients in the $1 \mathrm{GV} / \mathrm{cm}$ range [3], one to two thousand times higher than RF cavities. Since accelerating gradients are approximately $\sqrt{n} \mathrm{~V} / \mathrm{cm}$, where $\mathrm{n}$ is the plasma density, plasmas in solids can potentially deliver gradients 100 times higher than gas plasmas. For example, for $\mathrm{n}_{\mathrm{e}}=10^{22} / \mathrm{cm}^{3}$, the gradient would be $100 \mathrm{GV} / \mathrm{cm}$.

At the plasma densities required for acceleration there are severe material limitations. This has led to speculation about utilizing channeling [4] as an adjunct to solid state plasma acceleration [5]. Channeling could mitigate the problems and

p-Conf-01-029-E.dot submitted to World Scientific : 03/28/01 : 1:55 PM 
perhaps also introduce focusing to prevent beam blow-up from multiple scattering. At the intensities needed for solid state accelerators there will be significant channeling problems since the crystal lattice will be severely disturbed or vaporized. As the bunch intensity rises energy loss and plasma generation with the concomitant rise in crystal disorder will cause degradation in channeling [6] so that channeling might be quenched.

If channeling is to be considered for solid state acceleration more information is needed on the character and limitations of channeling under extreme conditions. Although existing channeling theory can serve as a guide, no channeling studies have been done under the non-equilibrium conditions that couple intense electron or laser energy into a crystal. Understanding of the behavior of solids under the conditions required for acceleration is in its early stages [7]. These processes are complicated but have been investigated in connection with terawatt laser technology and pellet fusion.

A systematic study of channeling with increasing bunch charge was carried out at Darmstadt [8] using the Darmstadt superconducting linac. A planar channeling experiment has also been done at relatively high bunch charge at Stanford on the Stanford Mark III accelerator [9]. Both groups investigated channeling radiation from electron beams in the 5-30 MeV energy region. The experiments were some orders of magnitudes away from the plasma acceleration regime.

The new Fermilab A0 Photo-Injector [10] produces a bunch intensity high enough to approach the plasma acceleration regime more closely. The accelerator typically operates with kinetic energy of $16.5 \mathrm{MeV}$. An experiment is now being carried out at the photo-injector to observe channeling radiation in this regime. Channeling radiation is studied as a function of electron bunch intensity to investigate whether it quenches as the bunch intensity is increased. If crystal disorder reaches the stage where channeling is quenched or extinguished the channeling radiation signal will diminish or disappear. A later stage of the experiment may attempt solid-state plasma acceleration although the available beam intensities are still far from those required for interesting acceleration.

\section{The Experiment}

Channeling radiation is straightforward to observe. Particles moving along a crystal plane or axis oscillate about the plane and radiate in the same way synchrotron radiation is produced in an undulator. The electron beam is deflected by a magnet after it passes through the crystal. The undeflected channeling radiation is detected by an x-ray detector. In the relativistic regime the "line energy" of the radiation [11] goes as $\mathrm{p}^{3 / 2}$ where $\mathrm{p}$ is the electron momentum. At A0 the channeling $\mathrm{x}$-ray energies are in the 10-200 keV range. Channeling X-rays are separated from other sources such as bremsstrahlung by scanning the crystal through the characteristic channeling angular distribution that has a width related to the Lindhard critical angle. The expected channeling radiation x-ray yield per electron for $\mathrm{Si}$ is on the order of $10^{-4}$.

p-Conf-01-029-E.dot submitted to World Scientific : 03/28/01 : 1:55 PM 
At A0 there are characteristically $5^{*} 10^{10}$ electrons in a bunch so that there are of order $5^{*} 10^{6}$ channeling $\mathrm{x}$-rays per bunch. These are concentrated in a cone that has an angular half width of $1 / \gamma$ or 30 milliradians. In a pico-second long pulse $10^{5}$ photons will strike a $125 \mathrm{~mm}^{2}$ detector $1.47 \mathrm{~m}$ downstream of the crystal.

Conventional single $\mathrm{x}$-ray detectors do not work in the extremely high $\mathrm{x}$-ray flux environment of the A0 photo-injector. Instead two other x-ray detector systems have been used. One employed an absorption-based, energy-resolved $\mathrm{x}$-ray detector (AberX) using a Ross filter system [12] and a lens-coupled scintillating screen-CCD system. This detector was developed by Freudenberger [13] to study its feasibility for mammography. The Ross filter technique takes advantage of the K-edge absorption of x-rays by thin metallic foils. A result has been obtained with that detector [14]. The second approach employs x-ray detectors made with Calcium Tungstate scintillation films monitored by photomultipliers (AberX-lite) and a Ross filter wheel. The experiment reported here using the second approach is continuing.

The A0 photo-injector normalized $\mathrm{rms}$ beam emittance with $6 \mathrm{nC} /$ bunch is $\varepsilon_{\mathrm{n}}=12 \mathrm{~mm} \cdot \mathrm{mrad}$ for typical conditions [15]. This is in line with simulations using the program HOMDYN [16]. The beam spot size at the crystal is typically $0.5 \mathrm{~mm}$ $(\sigma)$ so that the corresponding angular divergence is 0.7 milliradians. This is somewhat smaller than the axial channeling critical angle which is about $3 \mathrm{mrad}$ for the $\mathrm{Si}\langle 100\rangle$ axis at $\mathrm{A} 0$ energies. The bunch length for a $5 \mathrm{nC}$ bunch measured using a streak camera is typically $\sigma_{\mathrm{t}}=7 \mathrm{ps}$.

Figure 1 shows a schematic of the Fermilab channeling radiation experiment. This apparatus consists of a crystal mounted in a remotely controlled goniometer, a spectrometer magnet to deflect the electron beam, and an x-ray detector system. Beam current is measured with an integrating current transformer (ICT) and a Faraday cup. The $20 \mu \mathrm{m}$ thick, $25 \mathrm{~mm}$ diameter $\mathrm{Si}$ crystal was obtained from Virginia Semiconductor. The goniometer has two angular degrees of freedom, $\Theta_{x}$ and $\Theta_{\mathrm{y}}$. The goniometer design has been dictated by the requirements of the photoinjector dust-free, very high vacuum system.

The AberX-lite detector system has been calibrated in two separate ways. In one, the detector system was placed in a monoenergetic x-ray beam at the Argonne Advanced Photon Source with variable energy. The x-ray flux was measured by a calibrated ion chamber. The detector calibration extended over the 12-92 keV region. This gave the absolute response of the detector as a function of energy and the calibration of the Ross absorber system. The detector response was consistent with the foil thickness and the expected light yield of Calcium Tungstate.

p-Conf-01-029-E.dot submitted to World Scientific : 03/28/01 : 1:55 PM 


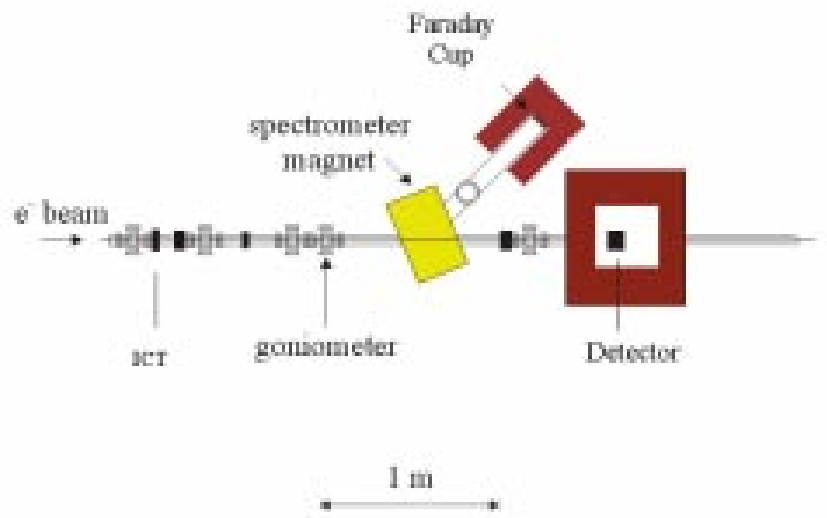

Figure 1.A0 channeling radiation apparatus.

In the second method the calibration is determined by integrating the x-ray yield over the bremsstrahlung spectrum and the channeling radiation spectrum determined from the $6.7 \mathrm{MeV} \mathrm{Si}<111>$ data of Genz et al. [17] extrapolated to 16.9 $\mathrm{MeV}$ and the $16.9 \mathrm{MeV}$ diamond $\langle 100\rangle$ data from Klein et al. [18] The response of the photomultiplier system can be determined from the bremsstrahlung yield. That in turn can be used to obtain the total number of channeling x-rays per electron. For this preliminary presentation the results from the two calibrations have been averaged and error bars shown that span the two methods. The Argonne light source calibration gives a lower response. Investigation of the differences between the calibrations is continuing.

Signal information from the Calcium Tungstate was collected using a digital oscilloscope. The scope integrated over the $\mathrm{CaWO}_{4}$ pulse, collected data from the integrating current transformer (ICT in Figure 1) and normalized the ICT signal to get the charge. The scintillation light time distribution for the $\mathrm{CaWO}_{4}$ has two time components; a short one with a time constant of 1.5 microseconds and a stronger one with 7.5 microsecond decay. The time structure of the A0 Photoinjector bunch train consists of a series of laser-driven pulses, each several pico-seconds long separated by one microsecond intervals. There is a background of dark current pulses coming at the $1.3 \mathrm{GHz}$ frequency of the RF. Typically the amplitude of a dark current pulse is $10^{-4}-10^{-5}$ of the amplitude of a laser-driven pulse. The relative amplitudes of the two sets of pulses can be controlled by changing the amplitude of

p-Conf-01-029-E.dot submitted to World Scientific : 03/28/01 : 1:55 PM 
the RF on the photo-injector RF gun and the laser pulse intensity. Since the principal aim of the experiment is to observe the channeling signal as a function of
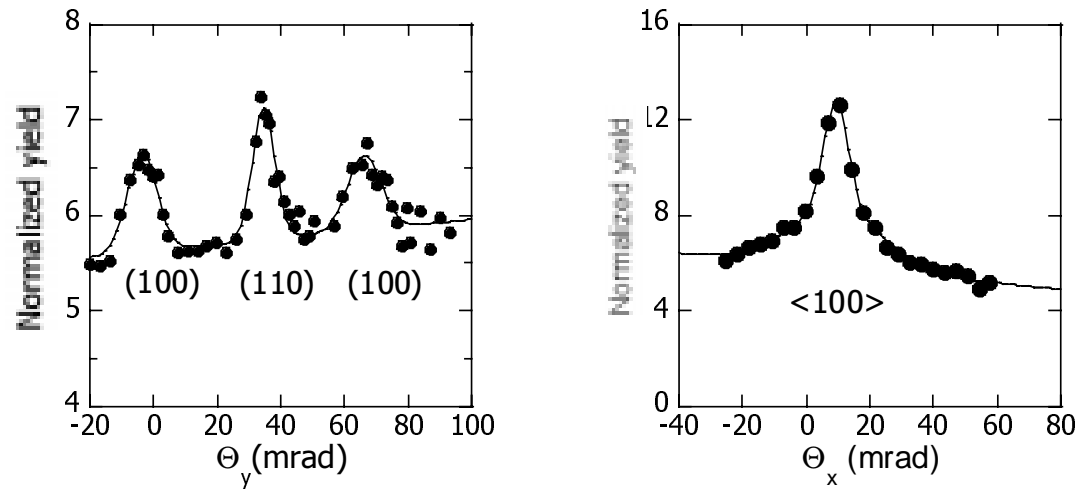

Figure 2. Planar and axial scans.

bunch charge it is desirable to operate over as wide a range of bunch charges as possible. This is done in two ways-by changing the laser intensity and by measuring the dark current yields. For the laser case care is taken to suppress the dark current and then subtract the residual by measuring the dark current just before the laser pulse and with no laser pulse. Typically measurements are made on the first laser pulse in a train and averaged over 10 cycles (A0 currently operates at $1 \mathrm{~Hz}$ ).

\section{Analysis}

Data is taken by first scanning the crystal through $\Theta_{\mathrm{x}}$ and $\Theta_{\mathrm{y}}$ to find a plane, an axis, or a random orientation of the crystal. The "no crystal" background is typically $17 \%$ of the yield on axis. Even a small electron or x-ray halo is multiplied significantly because the crystal holder is more than 100 times the thickness of the crystal. Most of the random background is due to bremsstrahlung in the crystal. Figure 2 shows typical scans through several planes and the $\langle 100\rangle$ axis. In the axial scan the crystal moves from the axis along a (110) plane because these planes are oriented in the horizontal and vertical directions. The peak is fitted with two co-located gaussians to account for the tilted planar portion. The $3.9 \mathrm{mrad}$ width $(\sigma)$ of the axial scan curve is consistent with the axial critical angle convoluted with the beam divergence and resolution due to other effects. The planar widths are slightly larger than expected. The ratio of the widths of the (100) and the (110) planes is consistent with

p-Conf-01-029-E.dot submitted to World Scientific : 03/28/01 : 1:55 PM 
the fact that the 45 degree planes are scanned diagonally. The ratio of planar height to axial height is consistent with theory and the results of earlier experiments.

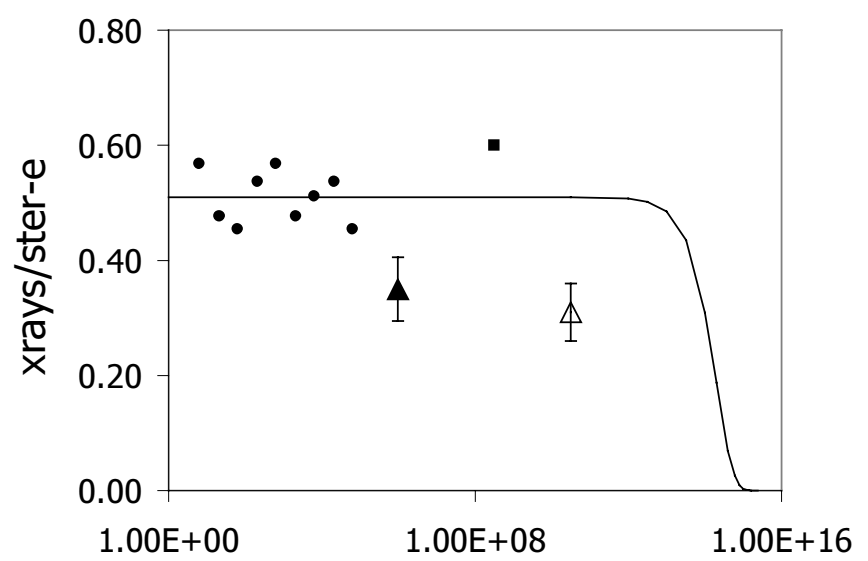

$\mathrm{n}$

Figure 3. Channeling radiation yield as a function of bunch charge.

Figure 3 shows the $\mathrm{x}$-ray yield per electron-steradian at $\mathrm{A} 0$ and for the earlier experiments as a function of electrons/bunch (n). The A0 results are represented by a laser driven axial channeling case with a bunch charge of $5 \mathrm{nC}$ (open triangle) and a dark current measurement with a bunch charge of $1.5^{*} 10^{-4} \mathrm{nC}$ (solid triangle). The dots are Darmstadt data taken at $5.4 \mathrm{MeV}$ for diamond $\langle 110\rangle$. The yield has been scaled by $\gamma^{5 / 2}$ to account for the yield energy dependence. This has been further multiplied by a factor of 8.62 to give the total x-ray yield rather than a $10 \%$ band. The square is a Stanford Si (110) planar point [19] taken at $30 \mathrm{MeV}$ and scaled to $16.9 \mathrm{MeV}$. The spectrum was integrated over all the x-rays and multiplied by 3.3 to account for the relative planar to axial yield. This assumption may overestimate the expected yield. It should be noted that both the Darmstadt and Stanford extrapolations introduce significant uncertainties by comparing different elements, orientations, and energies. The solid line is a schematic illustration of how the channeling radiation yield might quench with increasing bunch charge.

The A0 channeling radiation yield by itself is essentially flat over 4-5 decades while the combined Darmstadt-A0 set is flat to within $25 \%$ over ten decades. No evidence has been found of significant quenching of channeling at charge densities several orders of magnitude larger than in earlier experiments. This data is

p-Conf-01-029-E.dot submitted to World Scientific : 03/28/01 : 1:55 PM 
preliminary. More data is being gathered and the relationship between the two calibrations is still under investigation.

This experiment has reached bunch charges of up to $8 \mathrm{nC}$ in a beam spot with a sigma of $0.5 \mathrm{~mm}^{2}$ and a pulse length of $\sigma=7 \mathrm{ps}$. This corresponds to a current on the order of $1000 \mathrm{~A}$ and a current density of $10^{5} \mathrm{~A} / \mathrm{cm}^{2}$. The effective power density at A0 is typically $4 * 10^{11} \mathrm{~W} / \mathrm{cm}^{3}$. Achieving a $1 \mathrm{GeV} / \mathrm{cm}$ gradient could require power densities in the range of $10^{19}-10^{21} \mathrm{~W} / \mathrm{cm}^{3}$ so that the experiment is still a factor of $10^{7}-10^{10}$ away from where significant channeling acceleration could happen.

\section{Outlook}

\subsection{Solid State Plasma Acceleration}

Several approaches to solid state plasma acceleration have been discussed. One, particle wake field acceleration, uses a particle beam as a plasma driver. A laser beam can also be used to drive a plasma. Another approach is to use a side injected laser to avoid problems with pump depletion and particle dephasing [20]. Pump depletion is particularly troubling for the high plasma densities in solids. Approaches using laser beams are limited by the optical absorption depth for materials like $\mathrm{Si}$ and $\mathrm{Ge}$ as well as surface reflection.

As noted above, the A0 experiment is still far from the regime where significant solid state acceleration might occur. At A0 bunch compression can be used to reduce the bunch length to about $1 \mathrm{ps}$ and the spot size might be reduced by a factor of two so that it may be possible to go one order of magnitude further. A major problem for studying solid state acceleration is the bunch length. Getting into the plasma regime requires bunch lengths of $\mathrm{O}(10 \mathrm{fs})$. An approach to higher bunch charge densities is to go to a higher energy accelerator such as SLAC. There one might obtain beams with transverse sizes on the order of $5 \mu \mathrm{m}$ and bunch lengths of 50 fs [21]. This would increase the power densities by $10^{5}$ over the A0 result and approach much closer to the solid state plasma regime. Another approach would be to use a fairly modest electron beam coupled with extremely intense laser illumination.

Although the experiment is still far from the plasma regime in both current and pulse length, an initial search for plasma acceleration at A0 could be performed by assuming the beam itself would generate a plasma. The putative wake field could affect the tail end of the bunch so that it gained or lost energy. This could be observed by using the spectrometer magnet to look for a changing shape of the momentum distribution after the spectrometer with higher bunch intensities and with the crystal aligned for channeling or a random direction. The rms multiple scattering angle for the crystal produces a projected multiple scattering angle of 12

p-Conf-01-029-E.dot submitted to World Scientific : 03/28/01 : 1:55 PM 
mrad. This is equivalent to a momentum resolution of $0.6 \mathrm{MeV} / \mathrm{c}$. A plasma density of about $10^{17} \mathrm{e} / \mathrm{cm}^{3}$ would give a gradient of $0.3 \mathrm{GV} / \mathrm{cm}$ to give an equivalent energy gain in the $20 \mu \mathrm{m}$ crystal. This could be achieved with a side coupled laser with an intensity of $3 * 10^{15} \mathrm{~W} / \mathrm{cm}^{2}$. The A0 laser can reach $10^{8}-10^{9}$ watts $/ \mathrm{cm}^{2}$ for a $1.8 \mathrm{ps}$ pulse. Thus at present it is not possible to reach into the acceleration regime at $\mathrm{A} 0$ with the existing $\mathrm{A} 0$ laser.

\subsection{Other Applications}

Channeling radiation may be able to provide sub-picosecond x-ray fluxes that are higher than some other approaches now under development [22]. Sub-picosecond $\mathrm{x}$-ray processes are interesting because they can probe lattice vibration phenomena over a single oscillation. Existing synchrotron light sources are several orders of magnitude away from this possibility. Potential study topics include lattice vibration measurements, time-resolved chemistry, and 3-D motion of atoms. These types of experiments could not be done with the present pulse length at A0, which is currently longer than $1 \mathrm{ps}$.

\section{Acknowledgements}

The help of H. Edwards, R. Noble, J. Santucci (Fermilab), D. Haeffner, P. Lee, A. Mashayekhi, A. McPherson (Argonne), W. Hartung (Michigan State), J. Carneiro (Paris Sud-Orsay), M. Fitch (Rochester), and N. Barov (UCLA) is gratefully acknowledged.

*Operated by Universities Research Association, Inc. under contract No. DE-AC02$76 \mathrm{CHO} 3000$ with the United States Department of Energy

**Supported by BMBF contract number 06DA915I and by the DFG GK410

\section{References}

1. C. Clayton, , p. 13 and K. Nakajima, et al., p. 83 in Advanced Accelerator Concepts, eds. S. Chattopadhyay, J. McCullough, and P. Dahl, AIP Press C398, New York (1997).

2. See, for example, Advanced Accelerator Concepts, eds. S. Chattopadhyay, J. McCullough, and P. Dahl, AIP Press C398, New York (1997).

3. T. Katsouleas, p. 175 in Advanced Accelerator Concepts, eds. S. Chattopadhyay, J. McCullough, and P. Dahl (Lake Tahoe) AIP Press-CP398, New York (1997).

p-Conf-01-029-E.dot submitted to World Scientific : 03/28/01 : 1:55 PM 
4. For recent summaries of channeling see H. Andersen, R. Carrigan, and E. Uggerhoj, Nucl. Instr. and Meth. Channeling and other crystal effects at relativistic energy, B119 (1996) and R. A. Carrigan, Jr., p. 495 in Handbook of Accelerator Physics and Engineering, eds. A. Chao and M. Tigner, World Scientific, Singapore (1999).

5. P. Chen and R. J. Noble , p. 517 in Relativistic Channeling, eds. R. A. Carrigan, Jr. and J. A. Ellison (Plenum, 1987). P. Chen and R. Noble, p. 273 in Advanced Accelerator Concepts, eds. S. Chattopadhyay, J. McCullough, and P. Dahl, AIP Press C398, New York (1997).

6. R. A. Carrigan, Jr., p. 146 in Advanced Accelerator Concepts, eds. S. Chattopadhyay, J. McCullough, and P. Dahl (Lake Tahoe) AIP Press-CP398, New York (1997).

7. J. Davis, R. Clark, and J. Giuliani, Laser and Particle Beams, 13, 3 (1995).

8. W. Lotz, et al., NIM B48, 256 (1990).

9. C. K. Cary, et al., Nucl. Instr. and Meth. B51, 458 (1990).

10. J.-P. Carneiro, et al., proceedings of the 1999 Particle Accelerator Conference, New York (1999).

11. H. Genz, et al., Phys. Rev. B53, 8922 (1996).

12. See, for example, P.A. Ross, Phys. Rev. 28, 425 (1926), I. V. Khutoretsky, Rev. Sci. Instrum. 66, 773 (1995).

13. J. Freudenberger-Darmstadt thesis, "Roentgenstrahlung unter $180^{\circ}$ bei Elektronenenergien von 30 bis $87 \mathrm{MeV}$ am S-DALINAC, Aufbau eines Detektors fur Digitale Mammographie and Rontgenblitze sowie Test einer Rontgenlinse", Dissertation D17, Technische Universität Darmstadt (1999)unpublished.

14. S. Fritzler, "Untersuchung von Channeling-strahlung bei Hohen Ladungsdichten am Photo-injector des Fermi National Accelerator Laboratory”, Diploma Thesis, Technische Universität Darmstadt (2000)unpublished and J. Freudenberger et al.-to be published.

15. J.-P. Carneiro, et al., submitted to FEL 99 and J.-P. Carneiro, "Etude experimentale du photo-injector de Fermilab", dissertation-Universite de ParisSud, Orsay (in preparation).

16. M. Ferrario et al., Particle Accelerators 52, 1 (1996).

17. H. Genz, et al., App. Phys. Lett. 57, 2956 (1990).

18. R. K. Klein, et al., Phys. Rev B31, 68 (1985).

19. C. K. Cary, et al., Phys. Rev. B42, 7 (1990).

20. T. Katsouleas, J. M. Dawson, D. Sultana, and Y. T. Yan, IEEE Transactions on Nuclear Science, Vol. NS-32 (1985).

21. P. Chen-private communication.

22. R. A. Carrigan, Jr. and M. Kh. Khokonov, p. 516 in Quantum Aspects of Beam Physics, ed. P. Chen, World Scientific, Singapore (1999).

p-Conf-01-029-E.dot submitted to World Scientific : 03/28/01 : 1:55 PM 